\title{
Editorial
}

\section{Quality of Life of the Elderly in Japan}

The Welfare Ministry reported in 1988 that the average life expectancy of the Japanese population is 75.61 years in males and 81.39 years in females. The Japanese have now the longest life expectancy in the world. At present, the Japanese nation faces the aging of the society. This has never been experienced before and there is an urgency pressure to take medical and social measures to cope with the problem of the quality of life of the elderly.

Do today's elderly in Japan enjoy a good quality of life? Comparative studies between the elderly in Japan and those in the developed Western countries have indicated that the quality of life in the elderly in Japan is not consistently higher in terms of medical services and welfare, considering the powerful Japanese economy. In Japan, the adaptation of welfare policies and health management for the aging society has been extremely delayed.

With changes in the concept of family after the war and problems due to the acute housing shortage, the size of the average family has decreased, resulting in an increase in nuclear families. Old communities have also been disorganized in Japan, resulting in decreased opportunities for social exchange among the elderly. This is particularly true in cities, where the number of aged solitary people is increasing. Anxiety for living tends to be more marked in the elderly who do not live with their children. Nursing institutions are not adequate in number and quality. The number of bed-bound elderly people is expected to increase by about $50 \%$, exceeding 1 million in 2,000 A.D. and patients with senile dementia by about $50 \%$ to 100,000 . The current terminal care facilities for the elderly in Japan present many problems.

The primary cause of death in the Japanese is now cancer, followed in order by heart disease and cerebrovascular disease. Pneumonia and insenescence are also frequent causes. Multiple diseases tend to develop simultaneously in the elderly. One measure to prevent diseases is health management. Moderate exercise, balanced meals, adequate rest, a well-regulated life, and periodical health examinations are important. On examination, the elderly, even when seemingly healthy, frequently show multiple organ abnormalities, such as hypertension, arrhythmia, glucose intolerance, anemia, obesity, cataract, prostatic hypertrophy, spinal and articular deformity, and osteoporosis. To examine the elderly, it is necessary for doctors to comprehensively evaluate the patient.

The quality of life in the elderly differs depending on the individual, and a goal for meaningful life can not always be set. Since we have only one life, it is precious. Happiness in humans is the product of a healthy, rhythmic life and a sense of fulfillment.

There is a famous proverb by a Zen priest in China, "Every day is good day". If one can live every day as a good day that allows him to live fully but quietly in a serene state of mind, the end of his life may be wonderful.

We hope that the elderly may live a life in which their presence at home or in the society is significant in itself.

Shukuro ARAKI, M.D.

Professor and Chairman

The First Department of Internal Medicine

Kumamoto University Medical School

Kumamoto 